Letter to the Editor

\title{
Faculty outlook toward animal experiments in post-graduate medical education
}

Animal Experiments (AE) are fundamental in the training of almost all pharmacology-related post-graduate courses. ${ }^{1}$ However, research studies have questioned their human applicability, toxicological utility, and validity. Other limitations like their exorbitant costs, difficulty in procurement of animals, strict regulations, and reservations about animal ethics by animal rights organizations and students have also been observed. ${ }^{2}$ Moreover, newer opportunities and requirements in the pharmaceutical industry and clinical research organizations ${ }^{3}$ and recent trends in areas like pharmacoepidemiology, pharmacoeconomics, pharmacovigilance, rational pharmacotherapeutics ${ }^{1}$ have thrown AE out of focus.

Faculty perceptions have a strong influence on the curriculum followed and help in improvising the diverse post-graduate curricula followed in different medical colleges of India. ${ }^{1}$ Though studies have explored the students' and faculty perceptions of use of animals in undergraduate medical education, ${ }^{4}$ the faculty perceptions regarding use of animals in post-graduate training have not been looked into. Thus, it was deemed essential to assess the perceptions of the medical faculty about various aspects of $\mathrm{AE}$ in post-graduate medical education (PGME).

A cross-sectional survey was carried out among the pharmacology faculty in South India following institutional ethical committee approval. Faculty members from medical colleges, selected by convenience sampling method in the four southern states of India (Tamil Nadu, Karnataka, Kerala, Andhra Pradesh), took part in the study. The questionnaires with explanations of the objectives and instructions for filling were sent through post to the consenting faculty maintaining full confidentiality.

A structured questionnaire with closed-ended statements, determined through multiple focus group discussions with six pharmacologists (who themselves used AE in their teaching) and review of literature, was used to collect the data. Two professors in the subject, a medical education expert, and a sociopsychologist validated the content of the questionnaire and ensured that the statements in the questionnaire addressed all the study objectives.

After pilot testing on a group of five pharmacology faculty members, the face-validated questionnaire finally contained 27 statements. Some negative statements were introduced into the questionnaire and the statements were jumbled up in their order to circumvent answering bias.

The questionnaire was divided into the following five domains:

1. Advantages of $\mathrm{AE}$ in learning (ADV): seven statements about the usefulness, relevance, and worth of $\mathrm{AE}$ in learning.
2. Disadvantages of AE in learning (DIS): five statements regarding the drawbacks of $A E$ in PGME were included in this domain.

3. Logistics of conducting AE (CON): the time duration, economics, and other details about the conduct of AE were elaborated in six statements.

4. Faculty perceptions of student experiences with AE (PER): four statements focused on students finding these experiments stimulating, having lower exam stress, and being aware of learning objectives.

5. Alternatives to existing AE (ALT): five statements considered the knowledge about Government laws and characteristics of alternatives to existing $\mathrm{AE}$.

Categorical data were described as frequencies and percentage and analyzed by SPSS 19 version (IBM, Illinois, Chicago, USA).

Fifty-two faculty members (82.5\%) from eight private and seven state government institutions who responded to the questionnaire, had a mean age of $40.5 \pm 9.5$ years. The majority of the respondents were female, with less than 8 years of total teaching experience, holding an MD or a $\mathrm{PhD}$ degree and involved in post-graduate teaching.

At the time of the survey, the AE conducted in the participating colleges were: in vivo screening and toxicity in rat, mice, and rabbit (100\%) and graded dose responses in isolated tissues like frog rectus (100\%), rat colon (83\%), guinea pig ileum (50\%), frog heart (33.3\%), and rabbit aorta (16.7\%).

Around $75 \%$ of faculty members were in agreement with the advantages and logistics for conducting AE. However, opinions were divided on statements relating to disadvantages and other domains (Table 1). Majority of the faculty members perceived $\mathrm{AE}$ as a good learning experience by the students but disagreed that AE helped to lower student's stress. The faculty members were in favor of utilizing alternative for practical training but nearly half $(52 \%)$ of them were not willing to totally discontinue the $\mathrm{AE}$ in PG training.

AE contribute significantly in the training of post-graduates in the discipline of pharmacology. ${ }^{1}$ However, national legislations, student objections, animal right activists concerns, global awareness of three R's concepts (Replace, Reduce and Refine), ${ }^{5}$ and the attraction for the pharmaceutical industry/clinical research organizations $^{1,3}$ have made the use of animals in PGME debatable. The results of our study revealed that the majority of the participants support AE in PGME despite several shortcomings. A plausible reason for this may be that they perceive the need for post-graduates' training in $\mathrm{AE}$ for biomedical research and compliance with 
Table 1

Faculty members' perceptions regarding animal experiments in post-graduate medical education $(n=52)$.

\begin{tabular}{|c|c|c|c|c|c|}
\hline Domain & $\begin{array}{l}\text { S. } \\
\text { No. }\end{array}$ & Statement & $\begin{array}{l}\text { Agreement } \\
(\%)\end{array}$ & $\begin{array}{l}\text { Disagreement } \\
(\%)\end{array}$ & $\begin{array}{l}\text { Uncertainity } \\
(\%)\end{array}$ \\
\hline \multirow[t]{7}{*}{ ADV } & 2 & AE leave a long lasting impression of your discipline on students & $36(69.2)$ & $7(13.5)$ & $9(17.3)$ \\
\hline & 5 & AE help to encourage student centered education & $37(71.2)$ & $9(17.3)$ & $6(11.5)$ \\
\hline & 6 & AE improve dissection skills & $38(73.1)$ & $7(13.5)$ & $7(13.5)$ \\
\hline & 7 & AE encourage development of scientific research outlook & $44(84.6)$ & $3(5.8)$ & $5(9.6)$ \\
\hline & 15 & Students learn to handle live tissues through $\mathrm{AE}$ & $35(67.3)$ & $10(19.2)$ & $7(13.5)$ \\
\hline & 24 & AE provide an opportunity for deep learning rather than surface learning & $39(75)$ & $5(9.6)$ & $8(15.4)$ \\
\hline & 27 & AE seem relevant to a career in biomedical research & $43(82.7)$ & $2(3.8)$ & $7(13.5)$ \\
\hline \multirow[t]{5}{*}{ DIS } & 4 & $\begin{array}{l}\text { AE give too much of importance to factual learning (a method of learning which concentrates on memorizing } \\
\text { information) }\end{array}$ & $26(50)$ & $18(34.6)$ & $8(25.4)$ \\
\hline & 14 & AE cause unnecessary distress to animals & $39(75)$ & $9(17.3)$ & $4(7.7)$ \\
\hline & 16 & The same understanding of concepts of your discipline can be achieved without these experiments & $21(40.4)$ & $22(42.3)$ & $9(17.3)$ \\
\hline & 18 & The main objective of students to do $\mathrm{AE}$ is to pass University examinations & $17(32.7)$ & $28(53.8)$ & $7(13.5)$ \\
\hline & 26 & Students resist performing $A E$ & $16(30.8)$ & $24(46.2)$ & $12(23.1)$ \\
\hline \multirow[t]{6}{*}{ CON } & 1 & AE should be conducted in the program & $46(88.5)$ & $4(7.7)$ & $2(3.8)$ \\
\hline & 8 & The current use of animals for teaching purpose is ethically justified & $38(73.1)$ & $10(19.2)$ & $4(7.7)$ \\
\hline & 17 & The duration of time spent in $A E$ is too much & $23(44.2)$ & $24(46.2)$ & $5(9.6)$ \\
\hline & 19 & AE allow enhanced potential for repeatability of learning exercises compared with other alternatives & $14(26.9)$ & $29(55.8)$ & $9(17.3)$ \\
\hline & 20 & AE are economically viable & $19(36.5)$ & $21(40.4)$ & $12(23.1)$ \\
\hline & 23 & AE offer flexibility as to when and where experiments are conducted & $15(28.8)$ & $23(44.2)$ & $14(26.9)$ \\
\hline \multirow[t]{4}{*}{ PER } & 3 & Students find AE stimulating & $30(57.7)$ & $11(21.2)$ & $11(21.2)$ \\
\hline & 9 & Students are aware of the learning objectives for AE prior to the practical & $47(90.4)$ & $3(5.8)$ & $2(3.8)$ \\
\hline & 21 & $\mathrm{AE}$ result in lower student stress during exams & $12(23.1)$ & $31(59.6)$ & $9(17.3)$ \\
\hline & 22 & Demonstrations (of $A E$ in batches) are preferred rather than experiments done by students individually & $15(28.8)$ & $34(65.4)$ & $3(5.8)$ \\
\hline \multirow[t]{5}{*}{ ALT } & 10 & There are alternatives to $A E$ for practical teaching & $36(69.2)$ & $8(15.4)$ & $8(15.4)$ \\
\hline & 11 & Alternatives to $A E$ can achieve equivalent learning outcomes compared to $A E$ & $26(50)$ & $16(30.8)$ & $10(19.2)$ \\
\hline & 12 & $\begin{array}{l}\text { If alternatives (like computer assisted learning experiments, models) are available, AE should be totally } \\
\text { discontinued }\end{array}$ & $18(34.6)$ & $27(51.9)$ & $7(13.5)$ \\
\hline & 13 & If alternatives are available $\mathrm{AE}$ should be continued with reduction in no. or refinement of use of animals & $40(76.9)$ & $8(15.4)$ & $4(7.7)$ \\
\hline & 25 & Government laws have been framed with regard to use of animals in education/research & $49(94.2)$ & 0 & $3(5.8)$ \\
\hline
\end{tabular}

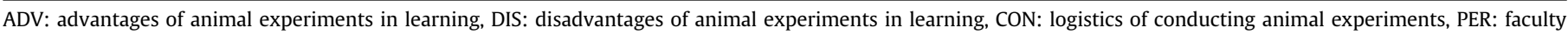

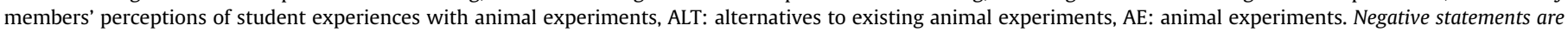
in italics.

regulatory toxicology requirements in the new drug development by the pharmaceutical industry.

In our study, the general agreement of the faculty members with all the statements in advantage domain reveals that the teachers support AE in PGME despite being aware of its potential disadvantages. Disagreement with statements that "students resist performing AE" but still perform them "to pass University Examinations" reveals the faculty members' perceptions about positive opinions of post-graduates toward $\mathrm{AE}$ (maybe due to its relevance in the post-graduates' future professional career). Ethical justification, post-graduates performing $\mathrm{AE}$ individually, and agreement with statements such as "students finding AE stimulating" and being "aware of learning objectives" again divulges their substantial support for animal use. They all seem to be aware of the alternatives to $\mathrm{AE}$ and the concept of three R's (Reduce, Replace and Refine) must be implemented.

Practical sessions in PGME fulfill learning outcomes like preparation-specific skills, animal handling, animal behavior, observational and surgical skills, research methodology, developing responsible attitudes toward animals etc. The alternatives do meet some of these objectives at least as effectively as live AE. Thus, introducing alternatives into PGME training necessitates the availability and familiarity with efficient alternatives. We advocate faculty training in physio-chemical methods and techniques utilizing tissue culture, microbiological system, stem cells, deoxyribonucleotide DNA chips, micro fluidics, computer analysis models, epidemiological surveys, and plant tissue-based materials which would minimize the number of animals used and circumvent some of the accepted disadvantages of $\mathrm{AE}^{6}{ }^{6}$ Furthermore, combinations of computer-aided learning techniques with manikins and chemical sensors can evade some of the disadvantages of alternatives and be a training tool which can completely replace few $A E$ in PGME.

The findings indicate that faculty members in South-Indian medical colleges support animal use in PGME in spite of being aware of their drawbacks and the availability of alternatives. In view of the global efforts to "Replace, Reduce and Refine" animal use, more awareness needs to be generated regarding the availability of alternatives to AE in PGME which will help our postgraduates to carry out scientifically valid animal-based research evading ethical issues.

We advocate the use of qualitative studies to evade the limitations of our questionnaire-based results. We also acknowledge the absence of randomization in our sampling procedure. Moreover, in view of the regional representativeness of the sample (from South India) and the small sample size, generalization of results to whole of the country may not be appropriate. Therefore, more studies on faculty perception from other parts of the country regarding $\mathrm{AE}$ should be performed before introducing the changes in post-graduate curriculum.

\section{References}

1. Dikshit RK. Postgraduate education in medical pharmacology. Indian J Pharmacol. 2007;39:171.

2. Hariharan TS. Need for changes in the practical pharmacology curriculum of medical undergraduates. Indian J Pharmacol. 2004;36:181.

3. Ghosh RK, Ghosh SM, Datta S. Training of postgraduate pharmacologists in India the need for alignment with the emerging roles in the pharmaceutical industry. J Postgrad Med. 2010;56:168-169.

4. Shehnaz SI, Sreedharan J, Mathew E, Gomathi KG, Sami Khan N. Willingness to spare animals in undergraduate medical education in Southern India: 
a preliminary questionnaire-based investigation. Altern Lab Anim. 2011;39: 557-566.

5. Guhad F. Introduction to the 3Rs (refinement, reduction and replacement). Contemp Top Lab Anim Sci. 2005;44(2):58-59.

Jayadevan Sreedharan

6. Arora T, Mehta AK, Joshi V, et al. Substitute of animals in drug research: an approach towards fulfillment of 4R's. Indian J Pharm Sci. 2011;73:1-6.

Research Division, Gulf Medical University, P.O. Box: 4184,

Ajman, United Arab Emirates

Syed Ilyas Shehnaz*, Anoop Kumar Agarwal, Mohamed Arifulla

Corresponding author.

Department of Pharmacology, Gulf Medical University, P.O. Box: 4184, Ajman, United Arab Emirates

26 July 2012

Available online 27 February 2013 